\section{Pseudoscience and antiscience}

\author{
Lewis Wolpert
}

The New Age: Notes of a Fringe Watcher. By Martin Gardner. Prometheus: 1988. Pp.273. \$19.95, £14.95.

IT Is, as we all know too well, very difficult to get a paper published in Nature. A lack of interest, or hint of doubt by a referee can sway the decision towards rejection. Yet in October 1974 this journal published a report that Uri Geller was able to divine the face of a dice a million times better than would be expected by chance. Reviewing the evidence, Martin Gardner argues that the original experiments were poorly controlled and, like so many other cases, were unreliably anecdotal. Nature's willingness to publish such a paper may be an extreme example of the desire among many people to believe in the paranormal and allow themselves to be tricked. "As all magicians know, physicists are the easiest in the world to be fooled by magic tricks."

In this series of essays, about half of which were published in the Skeptical Inquirer, Gardner, who is best known for his writing on mathematical recreations, devotes himself to debunking pseudoscience. Debunking is a pejorative term used by the defenders of the paranormal, but Gardner, following Stephen Gould, sees it as essential to the health of science. Many of the essays are devoted to exposing the fraud in claims, for example, for $\mathrm{N}$-rays, spiritualism and psychic surgery. The essay on the magic number 666 is great fun. With $A=100, B=101$, Hitler adds up to 666 .

A distinction should be made between pseudoscience and unconventional science. Gardner uses as an example the work of the astrophysicist Thomas Gold, whose career, he suggests, is a thousand times more interesting and significant than that of a crank like Velikovsky. Gold was brilliantly correct about pulsars but his views on the surface of the Moon, and more recently his non-organic theory of the origin of oil and natural gas, are totally unacceptable to most geologists. There is a natural and justifiable hesitation by scientists to accept unconventional ideas. But this should not be confused with the rejection of pseudoscience where there are neither data nor connections with the rest of science.

Examples of pseudoscience are Sheldrake's invocation of a quite new set of mystical forces, 'morphic resonance', to explain embryonic development, and claims for levitation or mind-reading. It is not just that the evidence is not available but there is only the flimsiest connection with current science. Perhaps a further distinction should be made within pseudoscience itself between those who are honourable and absurd, like Sheldrake, and those who are just fraudulent like Geller.

A threat to science comes less from the absurdities of the paranormal than from the sociologists of science. It is easy to dismiss a channeller who first made contact with Ramtha, who was born 35,000 years ago in the slums of Atlantis, when she put a model of the Great Pyramid on her head. Less easy to deal with, and more insidious, is the anti-science relativism of Feyerabend and Collins and Pinch. The latter, in their discussion of paranormal metal-bending have adopted a relativist position. Gardner, rightly, is shocked by their total indifference to whether the phenomenon exists or not. For them there is no rational means of evaluating conflicting claims. Just because there is no clear

way of demarcating between science and non-science should not prevent us from recognizing the absurd when we see it.

There are essays on perpetual motion, creationism and fundamentalist preachers, but as a whole, the book does not work. Though well written and witty, some of the pieces are too slight and the topics too parochial. Many of the targets are too easy. The book does not come to grips with the important issues as to why pseudoscience flourishes, whether it matters, and what we can do about it. In the end, stories about metal bending and Shirley MacLaine's channelling boyfriends become boring.

Lewis Wolpert is Professor of Biology as Applied to Medicine, Department of Anatomy and Developmental Biology, University College and Middlesex School of Medicine, Windeyer Building, Cleveland Street, London W1P 6DB UK.

\section{Cramp continues}

\section{Jeremy J.D. Greenwood}

Handbook of the Birds of Europe, the Middle East and North Africa: The Birds of the Western Palearctic. Vol. V, Tyrant Flycatchers to Thrushes. Editor-in-chief Stanley Cramp. Oxford University Press: 1988. Pp.1,075. £75, \$175.

STANLEy Cramp, who died last year, was a leading figure in British ornithology chairman of numerous committees, campaigner for conservation, driving force in establishing the Seabird Group, senior editor of British Birds. Amid all these activities, it is surely his editorship of 'BWP' that will ensure his place in history. There can be few serious ornithologists or respectable libraries in Europe that do not purchase each volume as it becomes available.

What can one say of the latest addition to such a well-known work, except that it is another element in a landmark in ornithology? The format of Vol. V is similar to that of earlier volumes, although, in keeping with the expansion of ornithological knowledge, there is 47 per cent more text per species than in Vol. I. The behaviour sections have continued to be more functional and less merely descriptive. The introductory material from Vol. I is presented again, with some revisions, especially concerning behaviour and habitat. There is still too little, however, on the methods used to obtain and present the information - for example, the difficulties of making repeatable measurements of birds and of estimating survival rates are not mentioned - and, overall, few of the criticisms made by reviewers of previous volumes have been met. Of course, those criticisms are minor in relation to the monumental significance of the work, and one can see that the failure to respond might be justified by the need to keep the presentation uniform throughout the volumes. But one hopes that they will be heeded when the second edition is produced.

My main personal disappointment is the unsatisfactory treatment of eggs. No standard deviations of measurements are given; nor are egg volumes. The expression 'calculated weight' is not explained and rates of loss of weight during incubation are not provided. Shape is described in terms that are said to be "selfexplanatory", a phrase which nearly always means that the writer is unwilling to be tied down to precise definitions. In a work such as this, terms should be defined clearly; even better would be to give, as well as length and breadth of eggs, the distance of the broadest point from the ends. The illustrations, too, are poor. Because of the wrong choice of background colour, some white eggs are almost invisible. And most of the photographs are badly illuminated: even the Dunnock's egg, in reality a heart-stoppingly bright blue, appears dull. Although a range of variants is shown for the eggs of most species, the relative frequencies of the variants are not.

At the present rate of progress, Vol. VII of BWP will appear 17 years after Vol. I, which will thus be substantially out of date. Could thought now be given to the idea of a continuing cycle of revision, one volume every three years say, so that new editions of each volume appear every 21 years? Such regular revision would be a great service to ornithology - and a continuing memorial to Stanley Cramp.

Jeremy J.D. Greenwood is Director of the British Trust for Ornithology, Beech Grove, Station Road, Tring, Hertfordshire HP23 5NR, $U K$. 